\title{
Zur Theorie \\ der für alle Massen doppelfokussierenden Massenspektrographen
}

\author{
Von Alfred Klemm \\ Aus dem Kaiser-Wilhelm-Institut für Chemie, Tailfingen \\ (Z. Naturforschy. 1, 137-141 [1946]; eingegangen am 1. März 1946)
}

\begin{abstract}
Es wird die Theorie von Massenspektrographen, bei denen für alle Massen Doppelfokussierung herrscht, auf Grund von Arbeiten aus der Mattauchschen Schule neu dargestellt. Die ionenoptischen Eigenschaften des homogenen Magnetfeldes werden rein geometrisch abgeleitet. Die Geschwindigkeitsfokussierungsbedingung ergibt sich durch Gleichsetzung der Geschwindigkeitsdispersionen des elektrischen und magnetischen Feldes. Die Beziehungen $s^{\prime \prime}=s a / a_{\mathrm{e}}$ für die Bildbreite und $d=a_{\mathrm{e}} /(2 s)$ für das Auflösungsvermögen werden unmittelbar abgeleitet. Die graphische Darstellung der Ergebnisse vermittelt einen guten Überblick der möglichen Anordnungen. Durch ein Zahlenbeispiel wird auf einen speziellen Fall mit $180^{\circ}$ magnetischer Ablenkung hingewiesen.
\end{abstract}

R. Herzog ${ }^{1}$, J. Mattauch und R. Herzog ${ }^{2}$ sowie R: Her zog und V. Ha k k haben eine allgemeine Theorie doppelfokussierender Massenspektrographen angegeben, die auch die Spezialfälle mit geradliniger Magnetfeldbegrenzung und Bildkurve umfaßt. Einer dieser Spezialfälle ist der bekannte Mattauch-Herzogscher Massenspektrograph, mit dem seit 1936 von $\mathrm{M}$ a t t a u $\mathrm{ch}$ und Mitarbeitern zahlreiche Untersuchungen durchgeführt worden sind, und den neuerdings $\mathrm{H}$. Ew ald technisch vervollkommnet hat. Da die Fälle geradliniger Polschuhbegrenzung und Bildkurve konstruktive und meßtechnische Vorteile bieten, und deshalb den Experimentator besonders interessieren, soll ihre Theorie in der vorliegenden Arbeit noch einmal für sich behandelt werden.

\section{Die Theorie}

Die Theorie ist zweidimensionale Ionenoptik erster Näherung. Das Ionenbündel geht erst durch ein elektrisches und dann durch ein magnetisches Feld (Abb.1). Denkt man sich den Objektspalt punktförmig und sieht man von den auszublendenden bzw. zu fokussierenden Nebenstrahlen ab, so laufen alle Ionen bis zum Eintritt ins Magnetfeld auf dem Mittelstrahl. Sie haben, wenn sie alle einwertig sind, die gleiche Energie, da sie im elektrischen Feld den gleichen Weg durchlaufen. Im

1 Z. Physik 89, 447 [1934].

2 Z. Physik 89, 786 [1934].

3 Ann. Physik 33, 89 [1938].

4 Z. Naturforschg. 1, 131-136 [1946].
Magnetfeld, das homogen angenommen wird, beschreiben sie Kreisbahnen, die der Gleichung

$$
H a=144 \mathrm{VMV}
$$

genügen. Dabei ist $H$ (Gauss) die Feldstärke, $a$ $(\mathrm{cm})$ der Krümmungsradius, $M(M E)$ das Isotopengewicht und $V$ (Volt) die Energie des Ions.

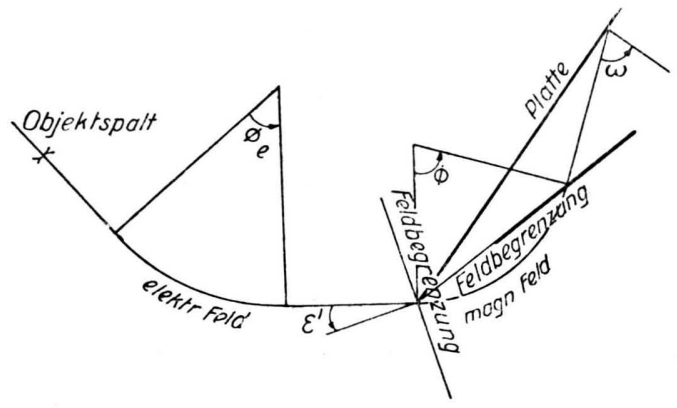

Abb. 1. Lage der Felder und Winkel.

Die hintere Magnetfeldbegrenzung und die photographische Platte liegen bei den betrachteten Anordnungen auf Geraden durch den Eintrittspunkt des Mittelstrahls in das Magnetfeld. Dann gilt für den Abstand $\rho$ eines Punktes auf der Platte vom Eintrittspunkt:

$$
\varrho=(\varrho / a)(144 \vee V M / H)
$$

Es sei $g^{\prime \prime}$ der auf einem Mittelstrahl gemessene Abstand des Brennpunktes des Magnetfeldes vom Austrittspunkt aus dem Magnetfeld. Wir werden zeigen [siehe Gl. (11)], daß $g^{\prime \prime}$ proportional zu $a$ ist und von der durch $\varepsilon^{\prime}$ gegebenen Richtung der 


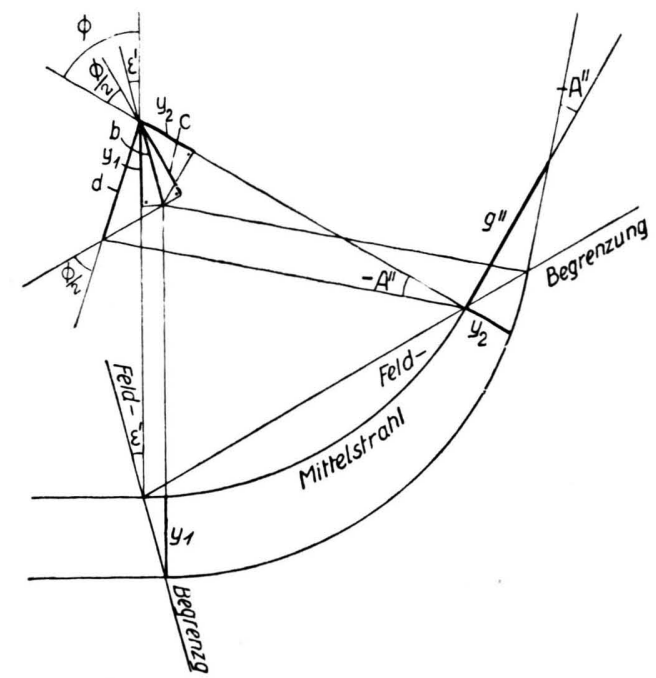

Abb. 2. Brennweite $g^{\prime \prime}$ des Magnetfeldes.

vorderen Magnetfeldbegrenzung abhängt. Die Brennlinie des Magnetfeldes ist also eine Gerade durch den Eintrittspunkt ins Magnetfeld, und es besteht die Möglichkeit, durch geeignete Wahl von $\varepsilon^{\prime}$ die Brennlinie des Magnetfeldes mit der photographischen Platte zur Deckung zu bringen. Bringt man nun den Objektspalt in den Brennpunkt des elektrischen Feldes, so entstehen auf der photographischen Platte Bilder des Objektspaltes, deren Lage von der Masse abhängt. Weiter werden wir zeigen, daß durch geeignete Wahl des noch frei verfügbaren elektrischen Ablenkwinkels $\Phi_{\mathrm{e}}$ erreicht werden kann, daß auch Strahlen, die den Objektspalt mit vom Mittelstrahl abweichender Geschwindigkeit verlassen, in den Bildern des Objektspaltes auf der photographischen Platte fokussiert werden.

Wir wollen nun die Beziehungen ableiten, die man zum Verständnis der hier besprochenen Anordnungen benötigt. Bei der Herleitung werden Produkte kleiner Größen stets vernachlässigt. Kleine Größen sind solche dimensionslose Größen, die beim Übergang von einem Nebenstrahl zum Mittelstrahl verschwinden.

Als erstes bestimmen wir die Lage des Brennpunktes eines homogenen Magnetfeldes mit scharfen Begrenzungen, wobei die hintere Begrenzung auf einer Geraden durch den Eintrittspunkt liegt. Wir betrachten einen Mittelstrahl mit dem Radius $a$ (Abb. 2) und fragen, wo ein Nebenstrahl mit gleichem Radius, der um $y_{1}$ gegen den Mittelstrahl parallel-verschoben ins Feld eintritt, den Mittel- strahl schneidet. Der Schnittpunkt ist der Brennpunkt. Wir bestimmen den Abstand $g^{\prime \prime}$ des Brennpunktes vom Austrittspunkt durch die Gleichung

$$
g^{\prime \prime}=-y_{2} / A^{\prime \prime} \text {. }
$$

Dabei ist $A^{\prime \prime}$ der Winkel, um den man den austretenden Nebenstrahl gegen den Uhrzeigersinn drehen muß, um ihm die Richtung des austretenden Mittelstrahls zu geben. $y_{2}$ ist der Abstand des Nebenstrahls rom Mittelstrahl beim Austritt. (Die Indices 1 und 2 bezeichnen Eintritt und Austritt.) Aus Dreiecken der Abb. 2 folgt:

$$
\begin{aligned}
y_{1} & =b \cos \varepsilon^{\prime}, \\
y_{2} & =b \cos \left(\Phi-\varepsilon^{\prime}\right), \\
c & =b \cos \left[(\Phi / 2)-\varepsilon^{\prime}\right], \\
c & =d \sin (\Phi / 2), \\
d & =-a A^{\prime \prime} .
\end{aligned}
$$

Durch Elimination von $b$ in (4) und (5) erhält man

$$
y_{2}=y_{1} \frac{\cos \left(\Phi-\varepsilon^{\prime}\right)}{\cos \varepsilon^{\prime}} \text {. }
$$

Weiter folgt aus (4), (6), (7) und (8) durch Elimination von $b, c$ und $d$ :

$$
A^{\prime \prime}=-\frac{y_{1} \cos \left[(\Phi / 2)-\varepsilon^{\prime}\right]}{a \cos \varepsilon^{\prime} \sin (\Phi / 2)} .
$$

Einsetzen von (9) und (10) in (3) ergibt

$$
\begin{aligned}
& \frac{g^{\prime \prime}}{a}=\frac{\cos \left(\Phi-\varepsilon^{\prime}\right) \sin (\Phi / 2)}{\cos \left[(\Phi / 2)-\varepsilon^{\prime}\right]}= \\
& =\left[\operatorname{ctg}(\Phi / 2)+\operatorname{tg}\left(\Phi-\varepsilon^{\prime}\right)\right]^{-1} .
\end{aligned}
$$

Als zweites berechnen wir die Geschwindigkeitsdispersion des Magnetfeldes und lassen zu diesem Zweck ein Ion auf dem Mittelstrahl vom Radius $a$ ins Magnetfeld eintreten, geben ihm aber einen Ra-

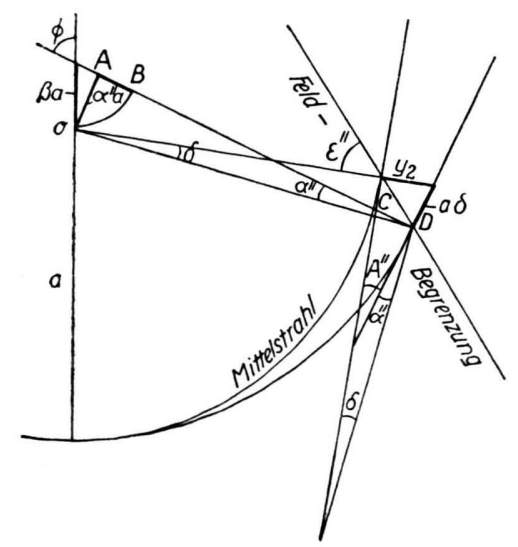

Abb. 3. Geschwindigkeitsdispersion des Magnetfeldes bei beliebiger hinterer Magnetfeldbegrenzung. 
dius $a(1+\beta)$, wo $\beta$ eine kleine Größe ist, und fragen nach dem Austrittswinkel $A^{\prime \prime}$ dieses Ions. Die Richtung der hinteren Magnetfeldbegrenzung wählen wir bei dieser Betrachtung beliebig. Aus Abb. 3 entnehmen wir für die Strecke $A B$ :

$$
A B=a \beta(1-\cos \Phi) \text {. }
$$

Aus $O C=A C=B D=a$ und $C D=y_{2}$ folgt $A B$ $=y_{2}$, also mit (12)

$$
y_{2}=a \beta(1-\cos \Phi) .
$$

Ferner entnimmt man der Abb. 3 die Beziehungen

$$
\begin{gathered}
A^{\prime \prime}=\delta+a^{\prime \prime} \\
a \delta=y_{2} \operatorname{tg} \varepsilon^{\prime \prime} \\
a \iota^{\prime \prime}=a \beta \sin \phi
\end{gathered}
$$

Einsetzen von (13), (15) und (16) in (14) ergibt

$$
A^{\prime \prime}=\beta\left[\sin \Phi+(1-\cos \Phi) \operatorname{tg} \varepsilon^{\prime \prime}\right] .
$$

Wir gehen nun zum elektrischen Feld über. Das Feld ist in einem sektorförmigen Gebiet vom Scheitelwinkel $\Phi$, in dem man die ebenen Polarkoordinaten $r$ und $\varphi$ benutzt. Die Feldstärke ist radial gerichtet und unabhängig von $\varphi$. Der Mittelstrahl tritt tangential ein und aus und beschreibt im Feld eine Kreisbahn vom Radius $a$. Man betrachtet dem Mittelstrahl benachbarte Bahnen, führt also ein

$$
r=a(1+z),
$$

wo $z$ eine kleine Größe ist. Die Feldstärke ist

$$
E=E_{0} a / r=E_{0}(1-z)
$$

und das Potential

$$
V=-a E_{\mathrm{u}} \ln (r / a)=a E_{0} z .
$$

Für Ionen, die sich auf Kreisen bewegen, gilt

$$
m v_{0}^{2} / r=-e E,
$$

wo $e, m$ und $v_{0}$ Ladung, Masse und Geschwindigkeit der Ionen sind.

Der Brennpunkt des elektrischen Feldes ist der Schnittpunkt eines um $y_{1}$ gegen den Mittelstrahl parallel-verschobenen eintretenden Nebenstrahles der Energie $m v_{0}^{2} / 2$ mit dem Mittelstrahl. Beim Eintritt in das Feld gewinnt jener Strahl die potentielle Energie

$$
e \mathrm{~V}_{1}=-e a E_{0} z_{1}=m v_{0}^{2} z_{1},
$$

wobei seine kinetische Energie um den gleichen $\mathrm{Be}$ trag kleiner wird:

$$
m v_{1}^{2} / 2=\left(m v_{0}^{2} / 2\right)-m v_{v}^{2} z_{1} .
$$

Seine Anfangsgeschwindigkeit im Feld ist also

$$
v_{1}=v_{0}\left(1-z_{1}\right) \text {. }
$$

Demnach lauten die Anfangsbedingungen für die Bahn im Feld:

$z=z_{1}, \dot{z}=0, \varphi=0, \dot{\varphi}=v_{1} r_{1}=\left(v_{0} / a\right)\left(1-2 z_{1}\right)$.

Wegen der Konstanz des Drehimpulses bei Wirksamkeit einer Zentralkraft folgt aus dem Anfangsdrehimpuls

$$
m r_{1}^{2} \dot{\gamma}_{1}=m a v_{0}
$$

für die Winkelgeschwindigkeit

$$
\dot{\varphi}=\left(v_{0} / a\right)(1-2 z) \text {. }
$$

Weiter liefert die Gleichgewichtsbedingung der Radialkräfte

$$
m \ddot{r}=m \dot{r} p^{2}+e E,
$$

wenn man $r, \dot{\varphi}$, und $e E$ durch $a, z, v_{0}$ und $m$ ausdrückt:

$$
\ddot{z}=-2\left(v_{0}^{2} / a^{2}\right) z .
$$

Mit Berücksichtigung der Anfangsbedingungen lautet die Lösung

$$
z=z_{1} \cos \left[\sqrt{2}\left(v_{0} / a\right) t\right] .
$$

Beim Austritt ist also der Abstand des Nebenstrahls vom Mittelstrahl

$$
y_{2}=y_{1} \cos (\sqrt{2} \Phi),
$$

und der Winkel zwischen Nebenstrahl und Mittelstrahl

$$
A^{\prime \prime}=\frac{1}{a} \frac{d y_{2}}{d \Phi}=-\frac{y_{1}}{a} \sqrt{2} \sin (\sqrt{\check{\Phi} \Phi)} .
$$

Aus $g^{\prime \prime}=-\left(y_{2} / A^{\prime \prime}\right)$ folgt für den Abstand des Brennpunktes vom Austrittspunkt

$$
g^{\prime \prime}=(a / \sqrt{2}) \operatorname{ctg}(\sqrt{2} \Phi) .
$$

Zur Berechnung der Geschwindigkeitsdispersion des elektrischen Feldes lassen wir ins elektrische Feld auf dem Mittelstrahl ein Ion eintreten, dessen Geschwindigkeit um $v_{0} \beta$ größer ist als die Geschwindigkeit von einem Ion gleicher Masse, das im Feld auf dem Mittelstrahl bleibt. Die Anfangsbedingungen für die Bahn im Feld sind dann:

$$
z=\dot{0}, z=0, \varphi=0, \dot{\varphi}=\left(v_{0} / a\right)(1+\beta) .
$$

Der Satz der Erhaltung des Drehimpulses liefert diesmal

$$
\dot{\varphi}=\left(v_{0} / a\right)(1+\beta-2 z) .
$$

Das gibt, in die Gleichgewichtsbedingung der Radialkräfte eingesetzt:

$$
\ddot{z}=2\left(v^{2} / \mathrm{a}^{2}\right)(\beta-z) .
$$


Die Lösung lautet

Demnach ist

$$
z=\beta[1-\cos (\sqrt{ } \cdot \mathbf{2}, \quad a)] .
$$

und

$$
y_{2}=a \beta[1-\cos (\sqrt{2} \Phi)]
$$

$$
A^{\prime \prime}=\frac{1}{a} \frac{d !_{2}}{d \Phi}=\beta \sqrt{2} \sin (\sqrt{2} \Phi) .
$$

Man macht nun die Richtung $A_{\mathrm{e}}{ }^{\prime \prime 5}$, mit der ein auf dem Mittelstrahl ins elektrische Feld eintretendes Ion der Geschwindigkeit $v_{0}(1+\beta)$ und der Masse $m$ (wobei $m v_{0}^{2} / a_{\mathrm{e}}=-e E_{0}$ ) aus dem elektrischen Feld austritt, gleich der Richtung $-A^{\prime}$, mit der ein Ion der Geschwindigkeit $v_{0}(1+\beta)$ und der Masse $m$ ins Magnetfeld eintreten muß, um auf dem zu $m v_{0}$ gehörenden Mittelstrahl aus dem Magnetfeld auszutreten. Dadurch erhält man Geschwindigkeitsfokussierung, denn bei der vorliegenden Art der Optik sind Parallelverschiebungen von Strahlen zwischen dem elektrischen und dem magnetischen Feld ohne Einfluß auf den Fokussierungsort. Die Geschwindigkeitsfokussierungsbedingung lautet also mit (17) und (39):

$\sqrt{2} \sin \left(\sqrt{2} \Phi_{\mathrm{e}}\right)=-\left[\sin \Phi+(1-\cos \Phi) \operatorname{tg} \varepsilon^{\prime}\right]$.

Dabei wurde die Umkehrbarkeit des Strahlenganges im Magnetfeld benutzt. Nun ist nach (11)

$$
g^{\prime \prime} / a=\left[\operatorname{ctg} \frac{\Phi}{2}+\frac{\operatorname{tg} \Phi-\operatorname{tg} \varepsilon^{\prime}}{1+\operatorname{tg} \Phi \operatorname{tg} \varepsilon^{\prime}}\right]^{-1} .
$$

Eliminiert man hier $t g \varepsilon^{\prime}$ mittels (40), so erhält man die Geschwindigkeitsfokussierungsbedingung in der Form

$$
g^{\prime \prime} / a=\sin \Phi+\frac{1-\cos \Phi}{\sqrt{2} \sin \left(\sqrt{2} \Phi_{\mathrm{e}}\right)} .
$$

Um die Breite des Spaltbildes $s^{\prime \prime} \mathrm{zu}$ berechnen, suchen wir den Bildpunkt eines Objektpunktes, der im Abstand $s$ (wo $s$ die Spaltbreite ist) vom Brennpunkt des elektrischen Feldes und vom Mittelstrahl liegt. Zur Bildkonstruktion wählen wir einen Strahl der Masse $m$, der den Objektpunkt parallel zum Mittelstrahl mit der Geschwindigkeit $v_{0}(1+\beta)$ verläßt, wo $\beta=s / a_{\mathrm{e}}$ und $m v_{0}^{?} / a_{\mathrm{e}}=-e E_{0}$ ist. Dieser Strahl behält in und nach dem elektrischen Feld seinen Abstand $s$ vom Mittelstrahl bei, denn er tritt tangential ein und hat im Feld die Geschwindigkeit $v_{0}$. Zwischen dem elektrischen und dem magnetischen Feld gehen wir nun zu einem Ion gleicher Masse und Geschwindigkeit über, das dort auf dem Mittelstrahl läuft. Auch dieses Ion gelangt zum

\footnotetext{
5 Größe des elektr. Feldes versehen wir von nun an mit dem Index e.
}

gesuchten Bildpunkt. Sein Impuls und damit Bahnradius ist prozentual um $\beta$ größer als beim Mittelstrahl. Es tritt daher im Abstand $s^{\prime \prime}$ parallel zum Mittelstrahl aus dem Magnetfeld aus, wo

$$
s^{\prime \prime}=a \beta=s a / a_{\mathrm{e}} .
$$

Auf der Platte erscheint das Spaltbild um 1/cos $\omega$ breiter.

Zur Berechnung des Auflösungsvermögens $d$ betrachten wir ein Ion der Masse $m(1+\gamma)$ und der Geschwindigkeit $v_{0}(1-\gamma / 2)$, das bis zum Eintritt ins Magnetfeld auf dem Mittelstrahl läuft. Dieses Ion besitzt den Impuls $m v_{0}(1+\gamma / 2)$, es tritt deshalb um

$$
s^{\prime \prime}=a \gamma / 2
$$

parallelverschoben gegen den Mittelstrahl aus dem Magnetfeld aus. Wenn $s$ die Objektspaltbreite ist und $\gamma$ so groß gewählt wird, daß die Verschiebung $a \gamma / 2$ gleich der Spaltbildbreite $s a / a_{\mathrm{e}}$ ist, dann ist $\gamma^{-1}$ das Auflösungsvermögen $d$. Es ist also

$$
d=a_{\mathrm{e}} /(2 s) \text {. }
$$

Der Einfallswinkel $\omega$ der Strahlen gegen die Normale der Bildgeraden ist gegeben durch

$$
g^{\prime \prime} \mid \alpha=[\sin \omega-\sin (\Phi+\omega)] / \cos \omega .
$$

\section{Die möglichen Anordnungen}

Die Lage des Brennpunktes auf einem Austrittsstrahl hängt von der Richtung $\varepsilon^{\prime}$ der vorderen Magnetfeldbegrenzung ab, und zwar nach der Beziehung

$$
g^{\prime \prime} / a=\left[\operatorname{ctg}(\Phi / 2)+\operatorname{tg}\left(\Phi-\varepsilon^{\prime}\right)\right]^{-1} .
$$

Dabei ist $\varepsilon^{\prime}$ der Winkel, um den man den ins Magnetfeld eintretenden Mittelstrahl entgegen dem Uhrzeigersinn drehen muß, um ihn mit der Normalen der vorderen Magnetfeldbegrenzung zur Dekkung zu bringen $\left(-\pi / 2<\varepsilon^{\prime}<\pi / 2\right)$. Die Bedeutung von (11) wird durch Abb. 4 veranschaulicht. Abb. 4 zeigt den Eintrittsstrahl, einen Kreis, sowie „Linien gleichen Eintrittswinkels“. Dort, wo der Eintrittsstrahl den Kreis berührt, sei der Eintrittspunkt ins Magnetfeld, und ein anschließendes Kreisstück sei eine Bahn im Magnetfeld. Legt man eine Tangente an den Kreis, so sei damit ein Ablenkwinkel $\Phi$ und ein Austrittsstrahl festgelegt. Der Schnittpunkt eines solchen in die Abb. 4 eingezeichneten Austrittsstrahls mit einer „Linie gleichen Eintrittswinkels"ist Brennpunkt des Magnetfeldes, wenn der Eintrittswinkel den an die Linie geschrie- 
benen Wert hat. In Abb. 4 entsprechen dem jenseits der gekrümmten Linie $s^{\prime}=\mp 90^{\circ}$ gelegenen Gebiet Strahlengänge, bei denen schon innerhalb des Magnetfeldes ein erster Brennpunkt liegt, und zwar in Abb. 4 an der mit dem betreffenden $\varepsilon^{\prime}$ bezeichneten Stelle des Kreises.

Ist durch Wahl von $\varepsilon^{\prime}$ für Richtungsfokussierung gesorgt, so kann durch geeignete Wahl des elektrischen Ablenkwinkels $\Phi_{\mathrm{e}}$ zusätzlich Ge-

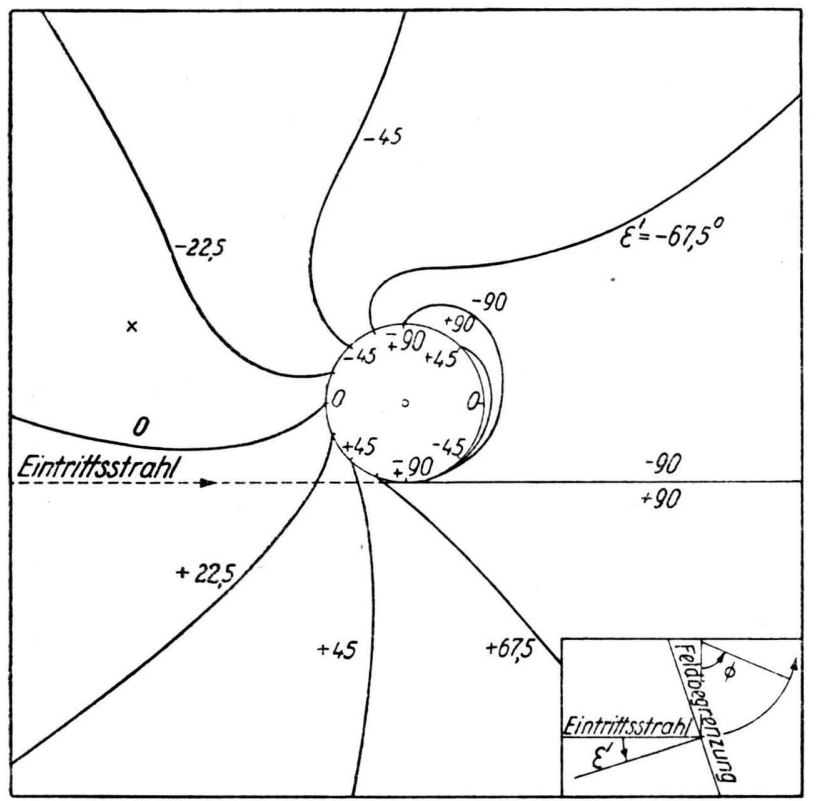

Abb. 4. Linien gleichen Eintrittswinkels $\varepsilon^{\prime}$.

schwindigkeitsfokussierung erreicht werden. $\Phi_{\mathrm{e}}$ hat den richtigen Wert, wenn es der Beziehung

$$
g^{\prime \prime} \mid a=\sin \Phi+\frac{1-\cos \Phi}{\sqrt{2} \sin \left(\sqrt{2} \Phi_{\mathrm{e}}\right)}
$$

genügt. Negatives $\Phi_{\mathrm{e}}$ bedeutet, daß die elektrische Ablenkung im Uhrzeigersinn erfolgt. Über die Größe von $\Phi_{\mathrm{e}}$ auf Grund von (42) bei allen möglichen Lagen der Brennpunkte hinter dem Magnetfeld gibt Abb. 5 Auskunft. In Abb. 5, die ebenso wie Abb. $4 \mathrm{zu}$ verstehen ist, sind der Eintrittsstrahl, eine Kreisbahn im Magnetfeld und „Linien gleichen elektrischen Ablenkwinkels" eingezeichnet. Der Schnittpunkt eines Austrittsstrahls mit einer „Linie gleichen elektrischen Ablenkwinkels“ ist Geschwindigkeitsfokus, wenn $\Phi_{e}$ den zur Linie gehörigen Wert hat. Außer dem Winkel $\Phi_{\mathrm{e}}$ gehört zu jeder Linie der Abb. 5 noch ein zweiter elektrischer Ablenkwinkel $\Phi_{\mathrm{e}}{ }^{\prime}$, der in Abb. 5 nicht eingetragen ist. $\Phi_{\mathrm{e}}{ }^{\prime}$ hat das umgekehrte Vorzeichen von $\Phi_{\mathrm{e}}$, und es ist $\left|\Phi_{\mathrm{e}}^{\prime}\right|=\left|\Phi_{\mathrm{e}}\right|+\pi / 2 . \quad \mathrm{Zu} \Phi_{\mathrm{e}}^{\prime}$ gehören Sirahlengänge, bei denen im elektrischen Feld ein Brennpunkt liegt. In dem Gebiet, das in Abb. 5 schraffiert ist, ist keine Doppelfokussierung möglich, weil es für die diesem Gebiet entsprechenden Wertepaare $\Phi$ und $g^{\prime \prime} / a$ keine $\Phi_{\mathrm{e}}$ gibt, die der Gl. (42) genügen.

Der Abstand $g_{\mathrm{e}}$ des Brennpunktes des elektrischen Feldes vom Eintrittspunkt in das elektrische Feld ist

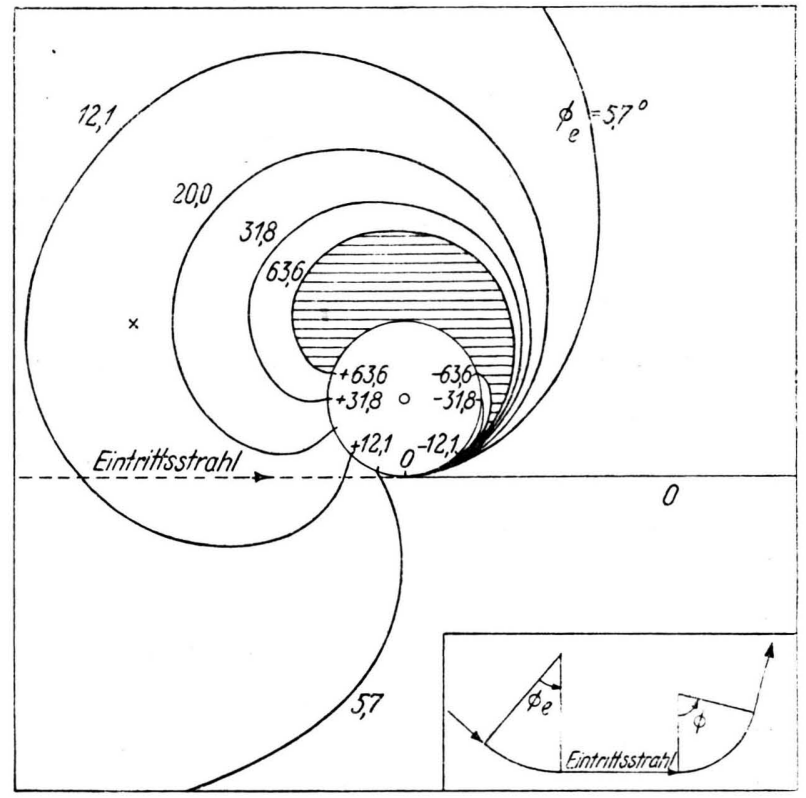

Abb. 5. Linien gleichen elektrischen Ablenkwinkels $\Phi$ ə

$$
g_{\mathrm{e}}=\left(\Phi_{\mathrm{e}} / \mid \Phi_{\mathrm{e}}\right)\left(a_{\mathrm{e}} / \sqrt{2} \operatorname{ctg}\left(\sqrt{2} \Phi_{\mathrm{e}}\right),\right.
$$

wobei $\Phi_{\mathrm{e}}$ negativ sein kann. Ist $g_{\mathrm{e}}$ negativ, so gibt es keinen Brennpunkt.

\section{Ein Zahlenbeispiel}

Es sei $\Phi=180^{\circ}$ und der Einfallswinkel der Strahlen auf die photographische Platte $\omega=60^{\circ}$. Dann ist nach (46): $g^{\prime \prime} / a=2 \sqrt{3}$. Setzt man dies, sowie $\Phi=180^{\circ}$ in (11) oder (41) ein, so erhält man $\operatorname{tg} \varepsilon^{\prime}=-(2 \sqrt{3})^{-1}=-0,289$, also $\varepsilon^{\prime}=-16^{0} 6^{\prime}$. Weiter ergibt das Gleiche in (42) eingesetzt: $\sin \left(\sqrt{2} \Phi_{\mathrm{e}}\right)=\sqrt{1 / 6}$, also $\Phi_{\mathrm{e}}=17^{\circ}$. In den Abb. 4 und 5 sind die diesem Falle entsprechenden Punkte durch Kreuze angedeutet. Mit $\sin \left(\sqrt{2} \Phi_{\mathrm{e}}\right)=\sqrt{1 / 6}$ wird nach $(33): g_{\mathrm{e}} / a_{\mathrm{e}}=\sqrt{5 / 2}=1,58$.

Hrn. Prof. J. Matta u ch danke ich für die Anregung dieser Arbeit und der Deutschen Forschungsge$\mathrm{meins} \mathrm{ch}$ a $\mathrm{t}$ für ein Stipendium. 